\title{
USING CAD AND FLOW SIMULATION FOR EDUCATIONAL PLATFORM DESIGN AND OPTIMIZATION
}

\author{
Cotet, C. E.; Popa, C. L.; Enciu, G.; Popescu, A. \& Dobrescu, T. \\ University POLITEHNICA of Bucharest, IMST Faculty, MSP Department, \\ 313, Splaiul Independentei, Sector 6, Cod 060042, Bucharest, Romania \\ E-Mail: costelemilcotet@gmail.com, laur.popa79@gmail.com, enciug@yahoo.com, \\ popescuadrian_c@yahoo.com, tibidobrescu@yahoo.com
}

\begin{abstract}
The main goal of the paper is to present how CAD and the simulation results of a virtual model were used to develop and adapt an educational platform to various manufacturing scenarios in an Industrial Logistics laboratory optimizing the performances in terms of productivity. Our paper is divided in two parts. The first part describes the design and development of an educational platform containing an AS/RS (automated storage/retrieval system) system and a RGV (Rail-Guided Vehicle). All the phases of the platform development are presented, starting with 3D modelling, and ending with the platform testing and its integration in a manufacturing cell. The second part demonstrates the platform performance diagnosis and optimization in different functional scenarios using material flow simulation. The problems that occurred (when the platform becomes part of different types of manufacturing architectures) are analysed using the simulation reports diagnosis and a new simulation validates the optimization solutions.

(Received in December 2014, accepted in May 2015. This paper was with the authors 1 month for 1 revision.)
\end{abstract}

Key Words: Simulation, Performance Diagnosis, Industrial Logistics, AS/RS System, Witness

\section{INTRODUCTION}

The material flow management using simulation is illustrated in this paper using an educational platform developed in the Industrial Logistic Laboratory of our university. The platform was made from scratch during the last two years. We have chosen to design our own platform and not to buy a readymade one because we wanted to involve our students from the Industrial Logistics specialization from the very beginning in this project for educational purposes (see Fig. 1).

The function of a material storage system is to store materials for a period of time and to permit access to those materials when required [1]. The Materials Handling Institute (USA) defines an automated storage/retrieval system (AS/RS) as a combination of equipment and controls, which handles, stores and retrieves materials with precision, accuracy and speed under a defined degree of automation [2]. Another definition can be the following: Automated Storage and Retrieval Systems (AS/RSs) are warehousing systems that are used for the storage and retrieval of products in both distribution and production environments [3]. Automated storage/retrieval systems are custom designed for each application, although the designs are based on standard modular components. The basic AS/RS consists of a rack structure for storing loads and a storage/retrieval mechanism whose motions are linear (x-y-z motions) [4].

Automated Storage and Retrieval Systems (AS/RSs) have been widely used in warehouses since 1950s to store and retrieve unit-loads without interference of an operator. The main advantages of AS/RSs are savings in labour costs and floor space, increased reliability, and reduced error rates [5]. We can find AS/RS systems in many domains such as: warehousing and distribution, manufacturing, automotive, pharmaceutical etc. 


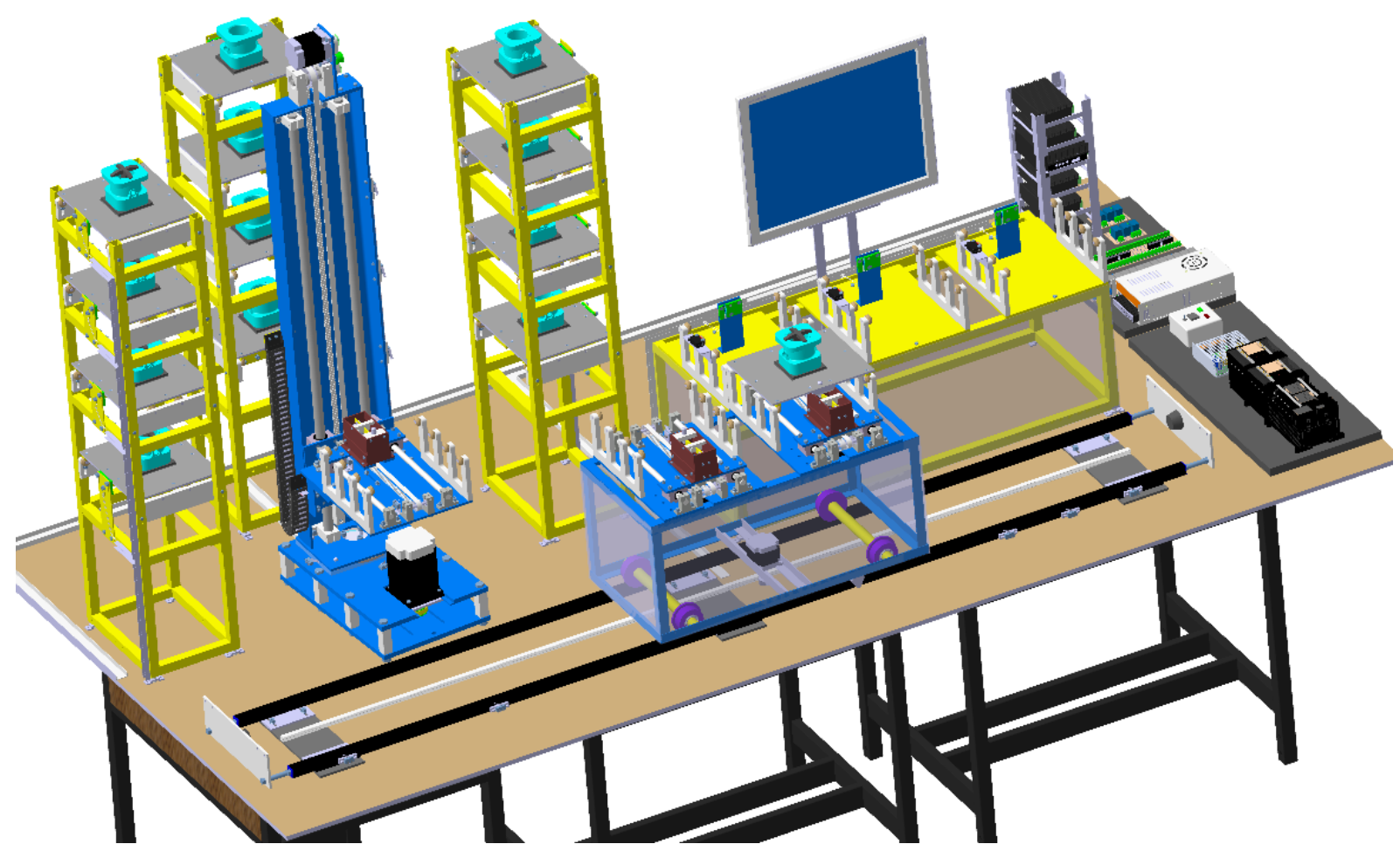

Figure 1: The 3D model of the educational platform in CATIA V5.

RGV (Rail-Guided Vehicle) are self-propelled vehicles guided by a fixed rail system. Vehicles operate independently and are driven by electric motors that in some systems can pick up power from an electrified rail [6]. There are two types of fixed rail system: Overhead monorail - suspended overhead from the ceiling and On-floor - parallel fixed rails, tracks generally protrude up from the floor. Also, routing variations are possible: switches, turntables, and other special track sections $[4,6]$.

In the initial system's version, the AS/RS educational platform was not designed to be mobile. It was later concluded that it would be useful for the logistics laboratory to be a mobile platform, so it can be relatively easily moved near various types of machine tools and robots from our department in order to perform more complex and diversified labs. The platform is in a continuous evolution, new ideas arise along the way, and we analyse the possibility and the necessity of implementing these new ideas. Small sessions were organized in order to stimulate the students' creativity and in these sessions they discussed the main options and proposals regarding the platform development.

\section{EDUCATIONAL PLATFORM DEVELOPMENT STAGES - MAIN COMPONENTS}

The platform design and development started from the need for such a system in the industrial logistics laboratory. The first step consisted in choosing the right system configuration, which would be designed, and several alternatives, technical characteristics and possible application that can be performed were proposed by our industrial partners, where our students are doing their internships. A first draft of the educational platform was sketched, and then was passed to the second stage where a 3D model for the main components of the platform was obtained. At this stage we worked in teams, each team designing a main 3D component. In the next section we will present the main components of the platform. 


\subsection{The AS/RS system}

The AS/RS system contains three modules: Base rotation module (BRM); Vertical translation module (VTM) and Palette transfer mode (PTM). The base rotation module is assembled with the vertical translation module, so the vertical translation module will be able to make a rotational motion for the transfer module positioning in the right shelves (see Fig. 2). The rotation of vertical translation module is made using a transmission. The V-belt is driven using a stepper motor (8 Nm). Dimensions: Height: $1020 \mathrm{~mm}$; Width: $245.5 \mathrm{~mm}$; Length: 394 $\mathrm{mm}$. The main components of the base rotation module are shown in Table I.

Table I: Base rotation module components.

\begin{tabular}{|c|l|c|c|c|}
\hline No. & \multicolumn{1}{|c|}{ Part name } & Material & Quantity & Obs. \\
\hline 1 & Spacer & Steel & 9 pcs. & Manufactured \\
\hline 2 & Bottom plate & Steel & 1 pcs. & Manufactured \\
\hline 3 & Upper plate & Steel & 1 pcs. & Manufactured \\
\hline 4 & Stepper motor & - & 1 pcs. & Acquired \\
\hline 5 & Hex cap screw M5×20 mm & Steel & 4 pcs. & Acquired \\
\hline 6 & Intermediate plate & Steel & 1 pcs. & Manufactured \\
\hline 7 & Hex cap screw M $8 \times 25 \mathrm{~mm}$ & Steel & 4 pcs. & Acquired \\
\hline 8 & Allen head bolt M6×30 mm & Steel & 3 pcs. & Acquired \\
\hline 9 & Shaft & - & 1 pcs. & Manufactured \\
\hline 10 & Stroke limiter support & Aluminium & 1 pcs. & Manufactured \\
\hline 11 & Stroke limiter & & 4 pcs. & Acquired \\
\hline 12 & Hex cap screw M10 $\times 100 \mathrm{~mm}$ & Steel & 9 pcs. & Acquired \\
\hline 13 & Timing Belts & - & 1 pcs. & Acquired \\
\hline
\end{tabular}

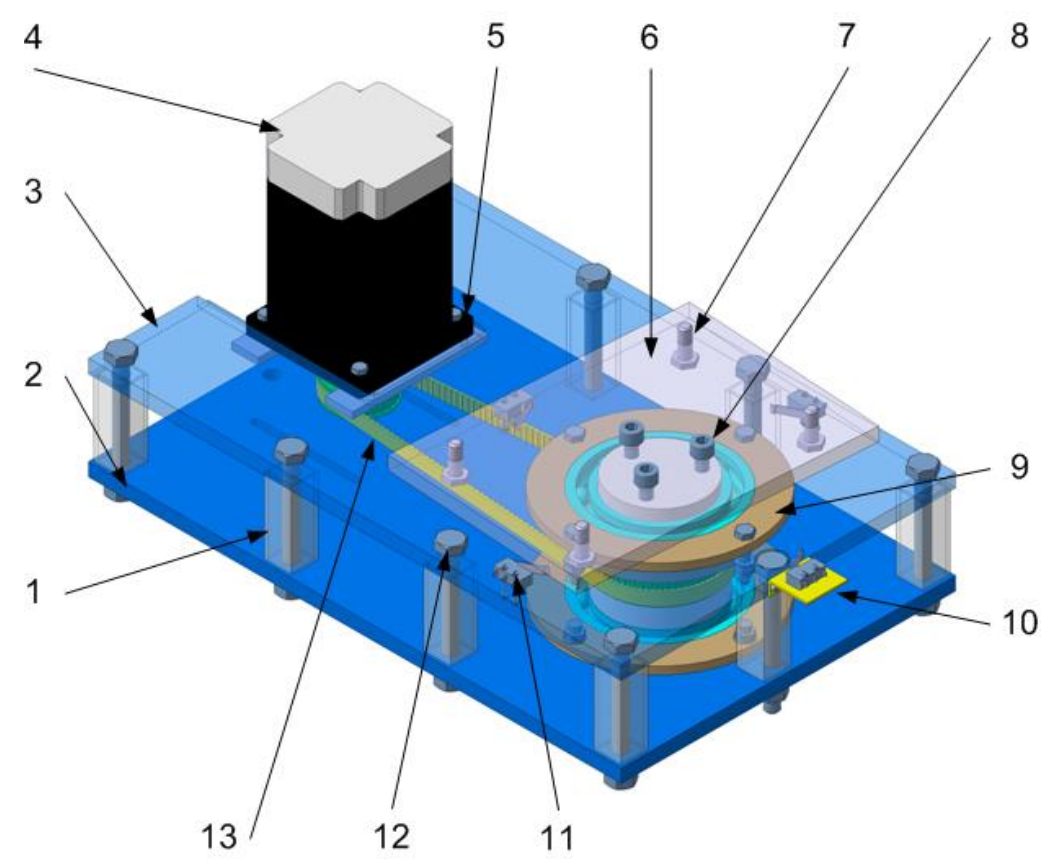

Figure 2: The 3D model of the base rotation module in CATIA V5.

\subsection{The storage system (rack)}

Dimensions: Height: 1000 mm; Width: 200 mm; Length: 270 mm 
The storage systems contains three racks, each with four shelves, arranged in a semicircular "U", shape, the opening is perpendicular to the translation direction of the AS/RS system.

\subsection{The RGV}

The RGV serves to transport the palettes between the intermediate storage stations and the AS/RS storage system. RGV is moving by using a rack-pinion mechanism. RGV has two transfer stations. Three photoelectric sensors are used in order to identify if a palette is present on the intermediary storage stations.

The RGV can reach a top speed of $234.375 \mathrm{~mm} / \mathrm{s}$ and a maximum stroke length of $1364.062 \mathrm{~mm}$. The maximum speed of the transfer system is $112.5 \mathrm{~mm} / \mathrm{s}$ and the maximum stroke length is $210 \mathrm{~mm}$. Dimensions: Height: $280 \mathrm{~mm}$; Width: $304 \mathrm{~mm}$; Length: $600 \mathrm{~mm}$.

\subsection{The educational platform drive and control}

To drive the subsystems described above were used stepper motors (see Fig. 3). Motor control was achieved with PLCs (Programmable logic controller) using specific drivers for selected stepper motors. In order to be taken on the transfer module, the palette needs a hook device, which was made by an electromagnet. On the AS/RS platform there are three such electromagnets. The electromagnets are controlled by PLCs through three relays. On the coil terminals a signal from the PLC is present for triggering the relay contacts. The relay contacts have a voltage of $24 \mathrm{~V}$ and $5 \mathrm{~A}$. When the relay coil is crossed by electric current it triggers two contacts of the relay closing an electrical circuit, so, a voltage also crosses the electromagnet coil, the coil electromagnet pulls inside a bolt, allowing pulling the palette through a lever.

The stroke limiters are positioned on the platform structure in order to control the RGV's position and the AS/RS position. The stroke limiters are located on the RGV's rail, respectively on the AS/RS storage system racks. When coming into contact with the stroke limiters the RGV and AS/RS system will stop if that limitation stroke limiter controls the program sequence.

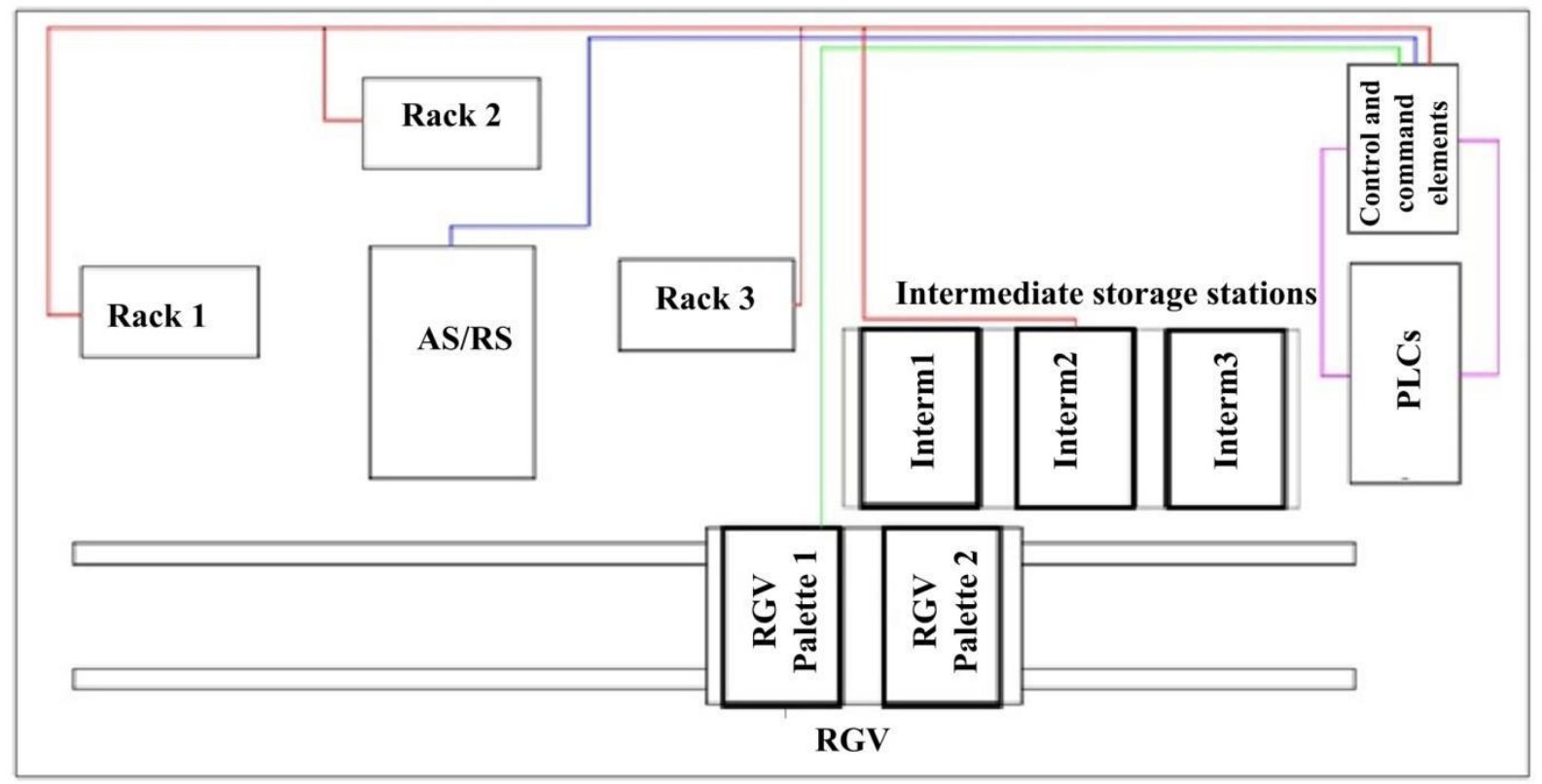

Figure 3: The educational platform drive and control sketch. 

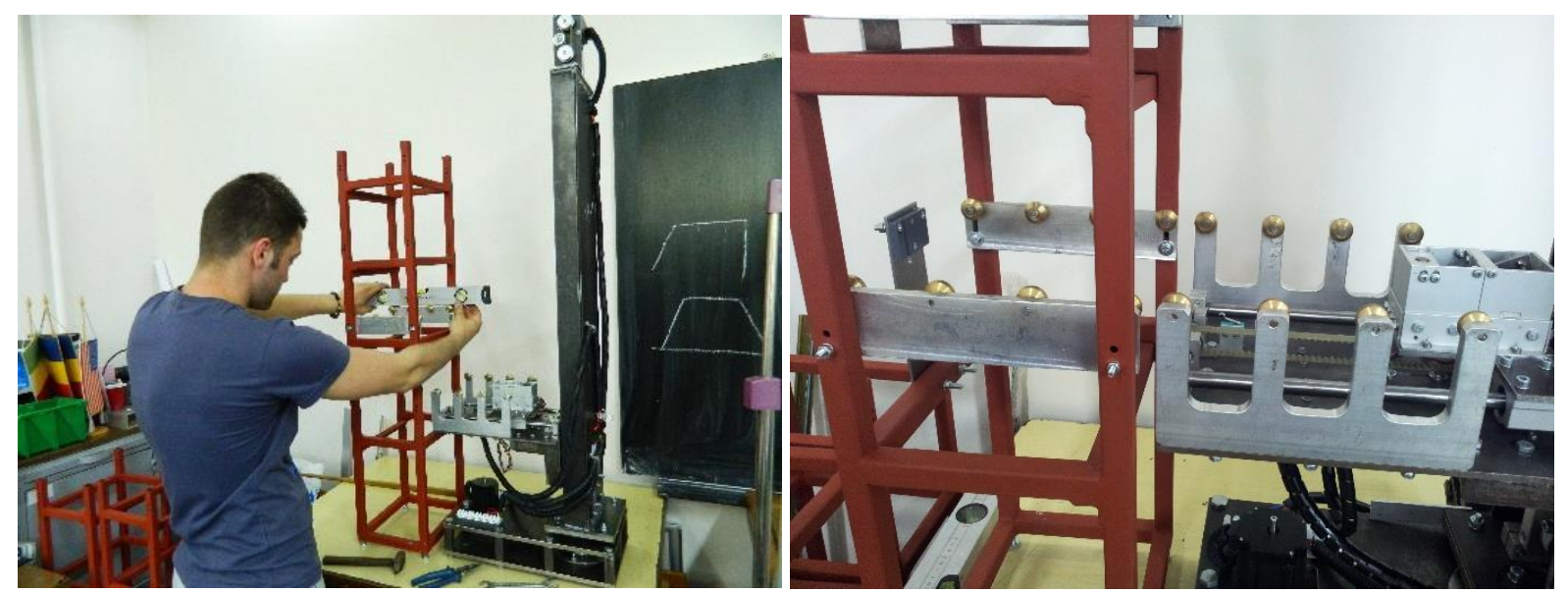

Figure 4: The assembly phase of the educational platform.

After the 3D modelling of the platform phase, the procurement process of some components followed, for motors, controllers, sensors and PLCs. Some part of the components was manufactured using the machine tools from the MSP department. Then the assembly of the main components began (see Fig. 4), and after that the final assembly of the platform was made including driving and control. In the next phase, the testing one, small changes and adjustments were made on the platform structure.

\section{OPERATING CYCLE PHASES}

The educational platform has a storage system with three racks, each rack has four storage stations, so the maximum storage capacity is 12 work pieces and parts. The AS/RS will take a work piece from the storage system and will transfer it to the RGV. RGV will transport the palette (with the work piece on it) to the end of the rail where are the three intermediate storage stations. Using stroke limiters and a cam positioned on it performs the RGV's precise positioning. After the RGV is positioned in front of the first two intermediate storage stations, the palette transfer is performed (transfer of palette with work piece from the RGV to the first intermediate storage station), in the same time, the palette with the part is transferred from the second intermediate storage station to the RGV (see Fig. 5).

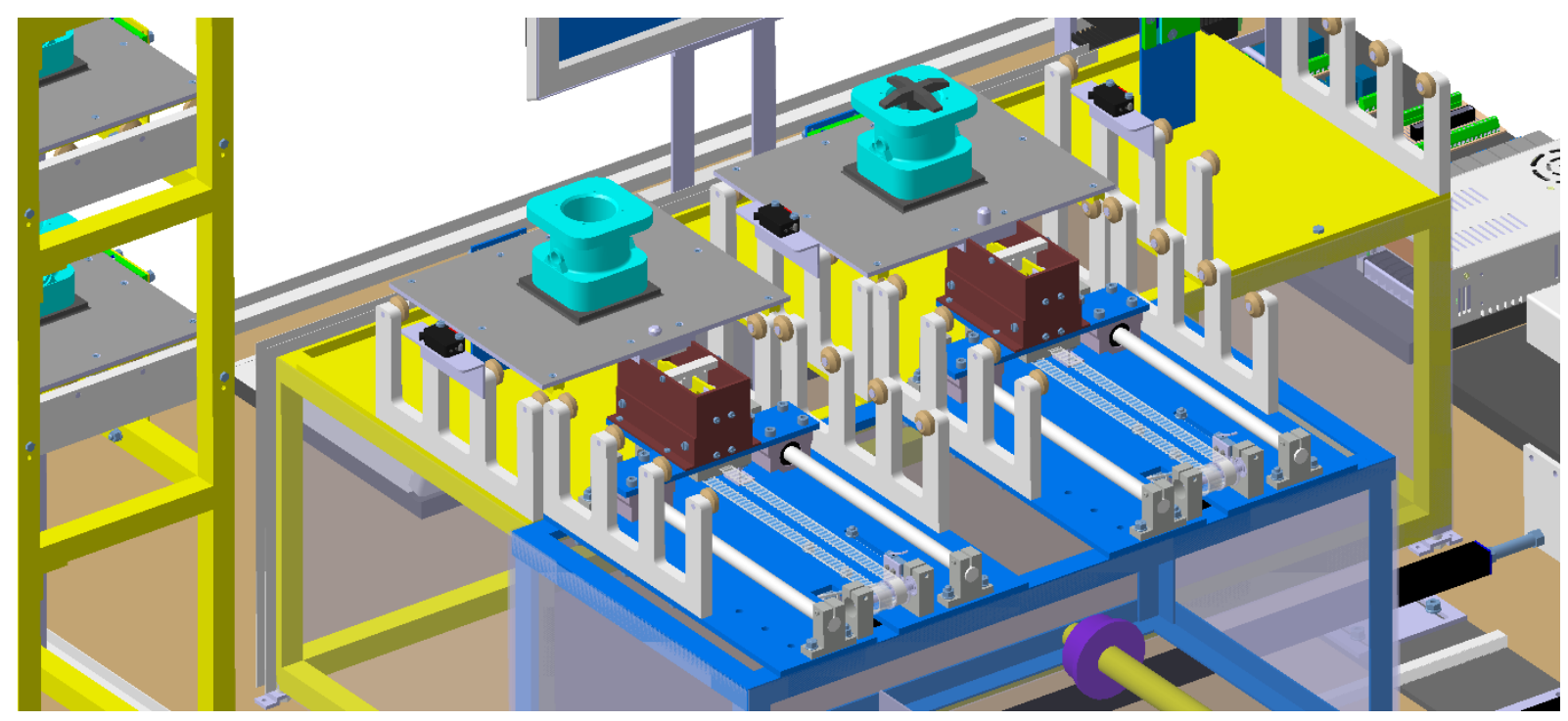

Figure 5: Transfer of the palettes from / to the intermediate storage stations. 


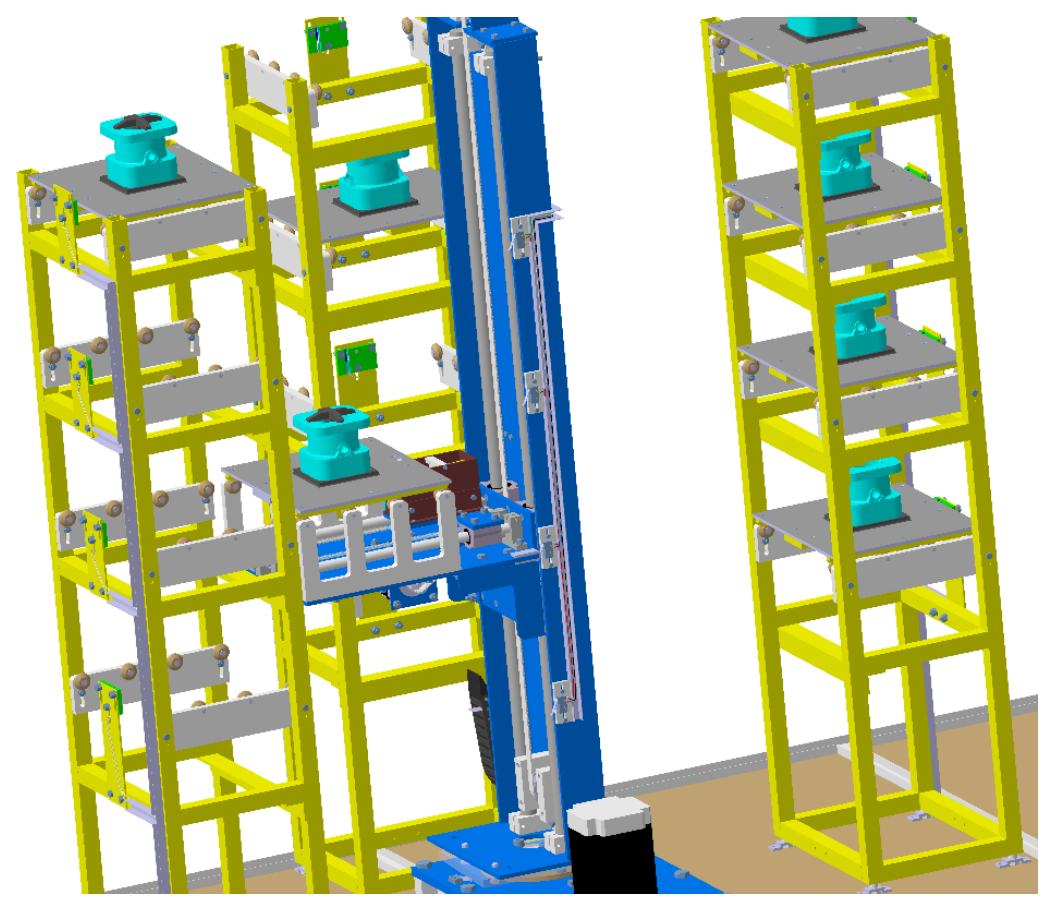

Figure 6: Transfer of the palettes from/to the storage system using AS/RS.

While the palettes transfer is made between the RGV and the intermediate storage stations, the AS/RS system is positioned in front of a storage station (part of the rack) and extracts a palette with a work piece to be transferred to one storage intermediates stations (see Fig. 6). According to the program made in CXprogramer the transfer system stops when a stroke limiter is driven. The blocking and the indexing of the palettes are made using magnetic sensor systems used to confirm the presence / absence of the palette in that position. Meanwhile the RGV having one of the palettes (with a part) is moving and is positioning itself in the front of the AS/RS system (see Fig. 7). After the transfer of the pallet with a work piece from the rack system, the AS/RS is positioned again in front of the RGV using the vertical translation module and the basis rotational module. The RGV is positioned in the front of the AS/RS system in order to be able to transfer the palette with a new work piece.

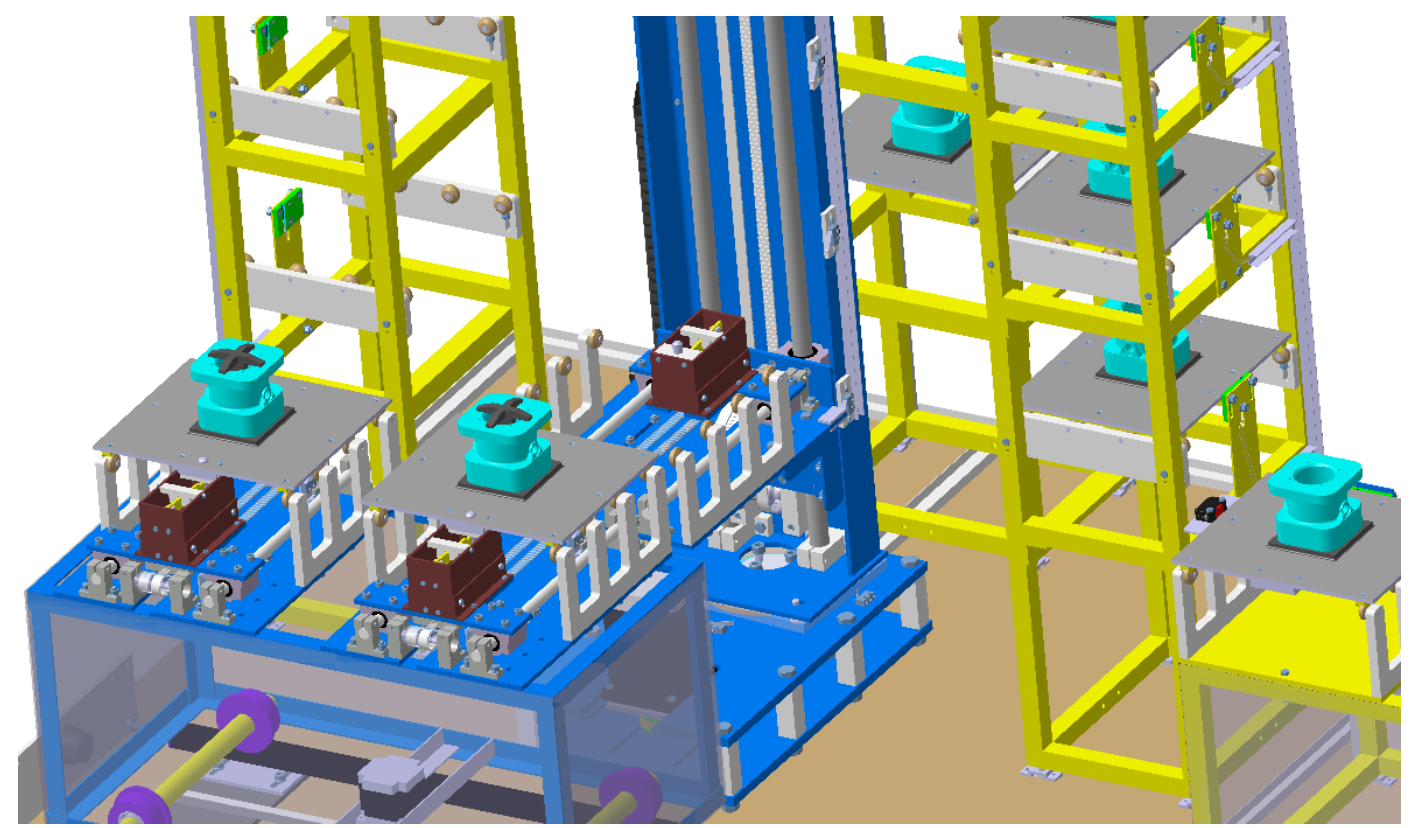

Figure 7: Transfer of the palettes from/to the RGV to the AS/RS system. 
After the transfer of the palette from the AS/RS transfer module to the first RGV's transfer system, the RGV is positioning for the second transfer system in the front of the AS/RS system. The AS / RS system is positioned with the first rack in order to store the palette with the part. On the last phase the RGV is transporting the pallet to the intermediate storage stations.

Using PLCs one makes the control of the motors, electromagnets, stroke limiters and sensors. Drivers supply the stepper motors for the main subassemblies of the platform. Two power supplies are connected with the electromagnets, the magnetic sensors and the stroke limiters.

\section{USING THE WITNESS SIMULATION SOFTWARE FOR THE SYSTEMS'S DIAGNOSIS}

Because the educational platform is mobile, it can be used in several case studies, so, the educational platform may be part of various architectures containing at least one work point (machine tool) and a transfer system (robot). A diagnosis for each system configuration is necessary. First, one has to make a material flow simulation of the new manufacturing architecture in order to identify the bottlenecks and long waiting times and to try to achieve an optimization solution for the system. The outputs of the optimized system represent the input data for the real system, for the command and control of the platform, for the robot and the machine tool programming.

Simulation allows the system optimization according to the machine tool type that is attached to the platform. The parameterization is made considering the work piece type to be processed, the processing time to obtain the part, the machine tool maintenance for batch of parts, the transfer time of the work piece to the machine tool and the transfer time of the part from the machine tool to the palette etc. In order to optimize the system, a separate diagnosis is made for each structural element, so that one can retain the optimum parameters that must be set for the studied system configuration. In this way one can make a quick setting of the platform structural elements using specific control and drive programs.

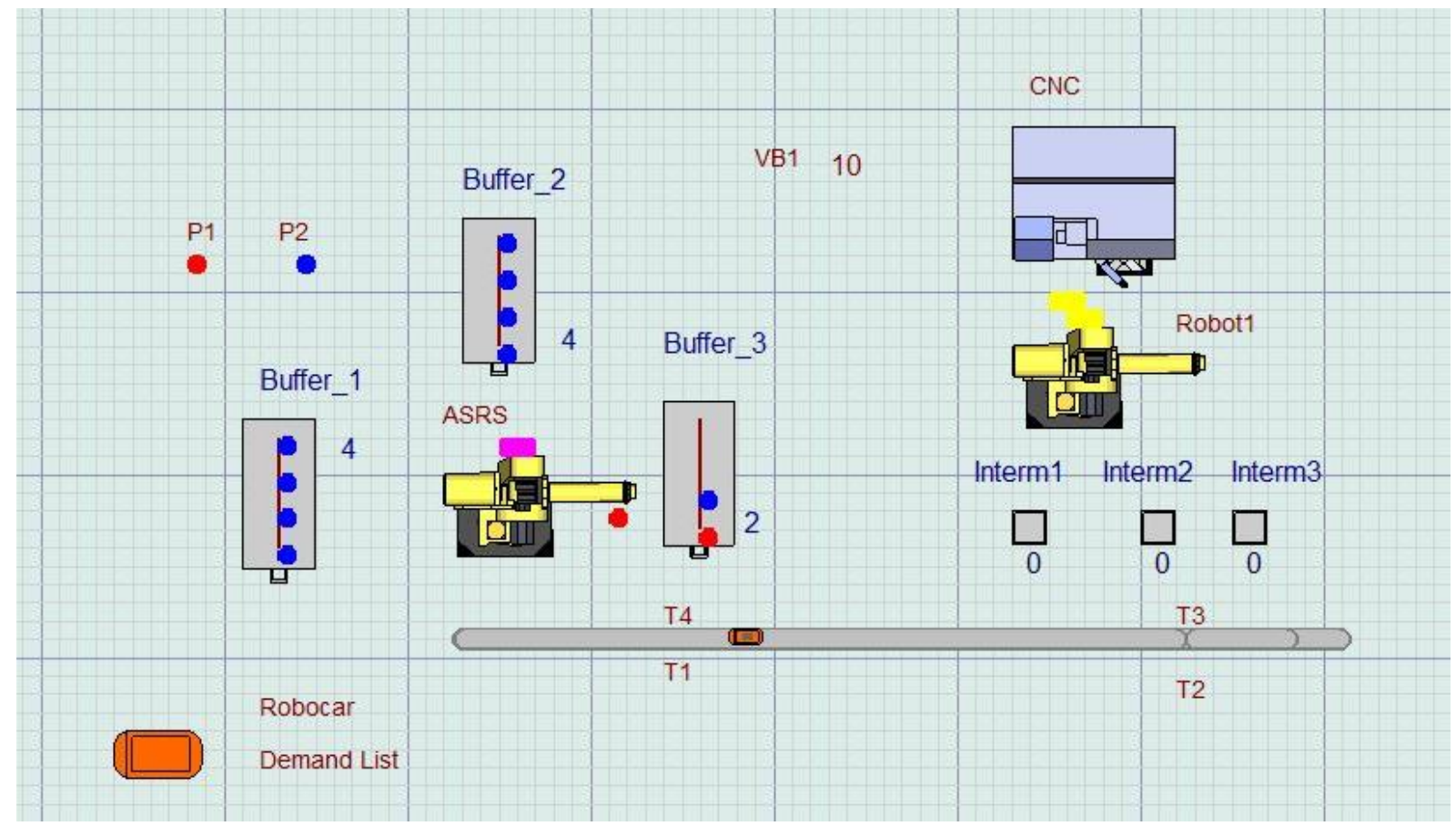

Figure 8: The platform during the simulation in Witness 13. 
Using the Witness 13 simulation software we can create a model for the whole manufacturing system including all the structural manufacturing elements: work points, transfer and transport systems, buffers, etc. (see Fig. 8).

For our case study we included the platform in a system configuration that contains a transfer system (robot) and a single work point (CNC machine tool). In order to establish the system's configuration we took into account the current trend in this area of using concentrate manufacturing systems.

We define then concentrate manufacturing systems as architectures based on a single work point surrounded \& assisted by transport, transfer \& deposit facilities. We defined diffused manufacturing systems as architectures with more than two work points connected by transport \& transfer systems and using deposits at local or system level. Diffused as well as concentrated manufacturing systems could be mass production, batch production and job shops [7].

All elements of the system have been modelled. The 3 racks, which correspond in the model with Buffer_1, Buffer_2 and Buffer_3, represent the storage system; each buffer has a storage capacity of 4 elements. The work pieces are represented by the P1 element and finished part by P2 element. We chose this way of representing to ease the system parameterization and monitoring. In fact the P1 elements (work pieces) are processed at the work of point (CNC) in order to obtain P2 elements (finished parts). We could also use one type of flow unit named $\mathrm{P}$ and after manufacturing, change its type attribute colour, but our chosen variant simplifies the parameterization of the structural elements and does not influence the final results.

The AS/RS system has been defined as a transfer element and the RGV as a transport element, which will move on the track (T1-T2-T3-T4). Interm1, Interm2 and Interm3 are the intermediate storage stations. Interm 1 is used to transfer the work piece, Interm 2 is used to transfer the finished part and Interm 3 is used only if one of the other two does not work or is blocked.

The parameterization process is based on the following constraints: time required to manufacture the part at CNC, transfer times between $\mathrm{CNC}$ and one of the three intermediate storage stations. The remaining elements are parameterized in order to meet these conditions and to determine an optimized version of this system configuration.

For a designed manufacturing architecture it is always useful to simulate the material flow conduct before applying our design into practice in order to avoid potential flow concentrators (bottlenecks) generating low productivity or even blockage [8].

Flow concentrators also generate under utilization of the structural elements of the manufacturing system leading to financial loss [9].

After the material flow simulation one can analyse the reports of all the structural manufacturing elements and can identify eventual bottlenecks where the material flow is slowed or blocked.

We defined a variable VB1 to count the number of parts machined on CNC in a time period. Thus we can see the productivity increase between different versions of the system parameterizations. In our case the variable will indicate 10 parts machined on CNC, it can be seen that the storage system are present 9 , the tenth is at RGV in order to be transported. There are two work pieces, one is in the storage system, and the other has already been taken by the AS/RS system to make the transfer because the RGV is approaching to the transfer position.

After the first simulation, we obtained 12 parts and some problems have been identified at the work point $(\mathrm{CNC})$ : the waiting time represents $40 \%$ of the total time, and the processing time represents $60 \%$ of the total time (see Fig. 9). 


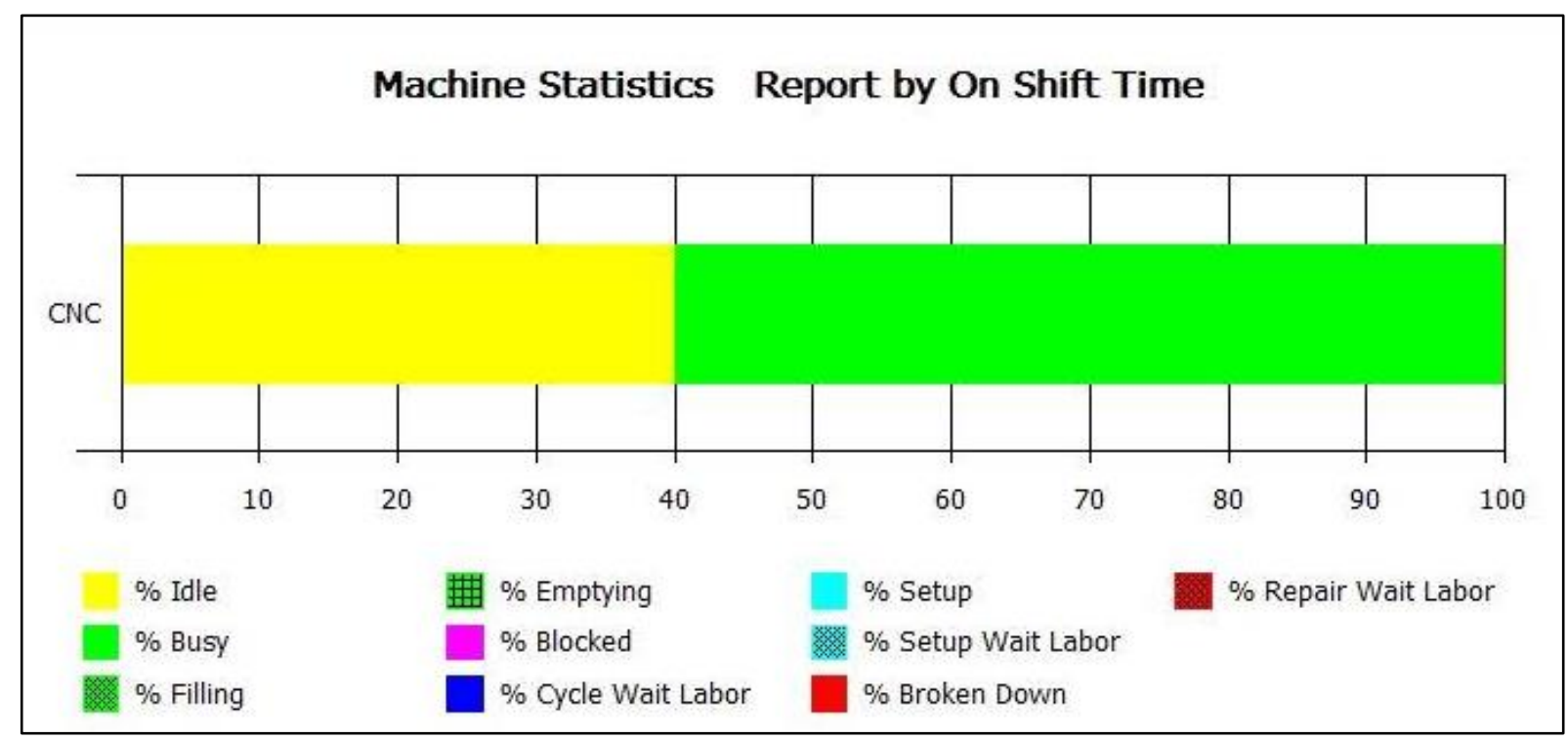

Figure 9: Report regarding the activity for machine CNC.

We can observe that on the intermediate storage station Interm1 were transferred 12 work pieces, which were taken by the Robot 1 transfer system. The average storage time was 10 seconds per each element. The report of Interm 2 storage station is identical to Interm1. Interm3 was not used because there aren't bottlenecks on Interm1 and Interm2 (see Fig. 10).

The RGV's speed is adjustable and it can be set according to the system requirement. However we cannot set values below a certain limit. It is preferable that the RGV remains stationary in front of the transfer system than to move with a very low speed.

During the optimization process we tried that the CNC and the RGV wait for parts and work pieces as little as possible. We tried to avoid the case in which the RGV would carry more work pieces to the intermediate storage station and to generate bottlenecks.

The CNC waiting time is related to the total simulation time and varies depending on the processing time. If a part needs a long term to be manufactured, for example the CNC machine needs 300 seconds in order to process the parts; the total waiting time decreases in percentage terms.

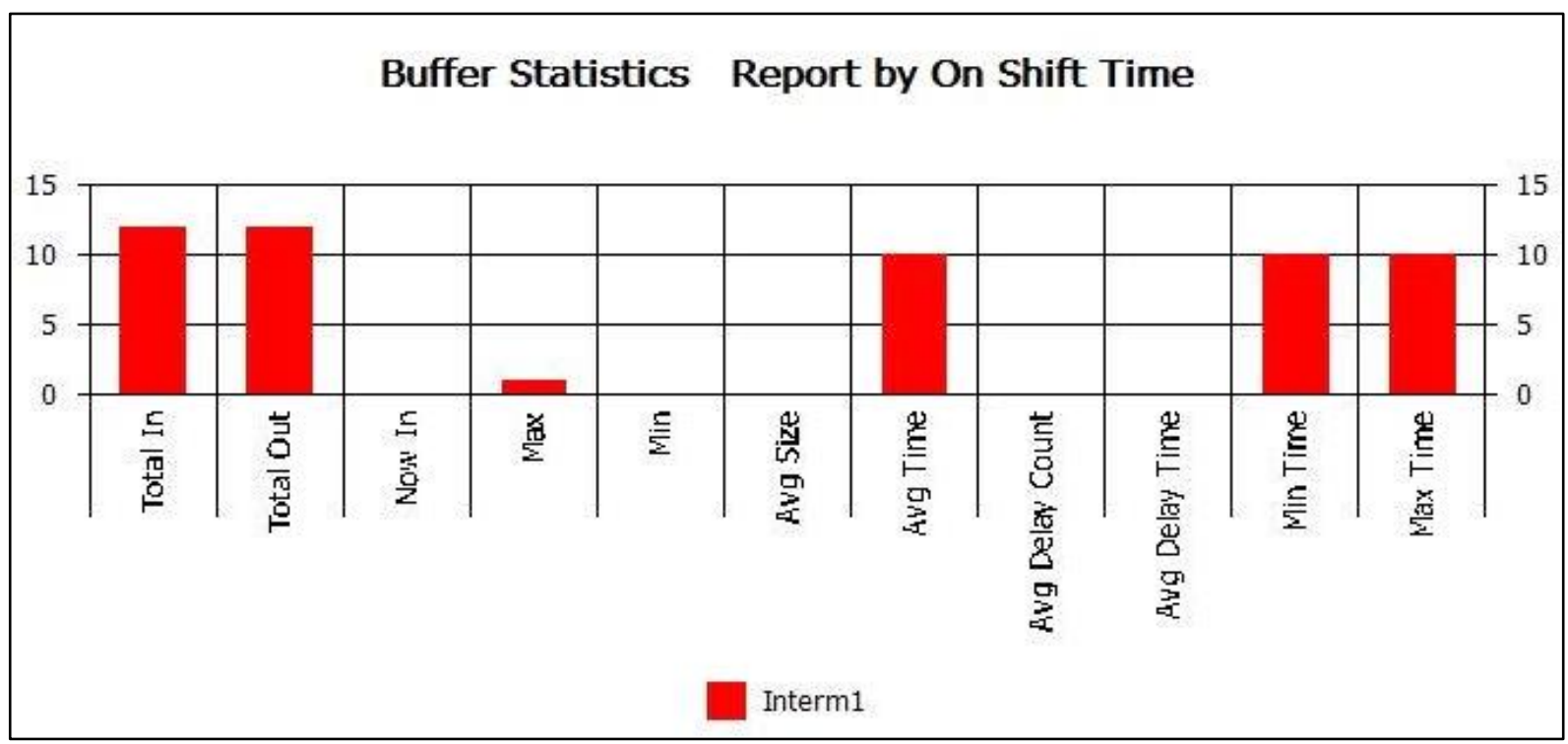

Figure 10: Report regarding the activity for Interm_1 storage station. 
If a part needs a short term to be manufactured, for example the CNC machine needs 180 seconds in order to process the parts, the waiting time will increase because there are maximum values for the parameters used for the rest of the elements (transport, transfer etc.) even if we push the components parameterization to the limits. The main idea is to find the optimum solution for requested processing time.

Several simulations were performed in order to optimize the system by changing the structural elements parameters. The biggest productivity increase version was kept, without bottlenecks and large waiting times. We tried to reduce the waiting time for the intermediate storage stations: once the transfer of the palette from the RGV is complete, the transfer of the work piece is done, respectively, once the part was transferred to Interm 2 palette, the palette is transferred to RGV. The report regarding the activity of CNC machine after the system optimization shows improvements: the waiting time represents $19 \%$ of the total time, and the processing time represents $80 \%$ of the total time (see Fig. 11).

Starting from the modelled virtual system, various system parameterization versions were tested; system's simulations were performed and the best system version identified [10]. The parameters resulted from the simulation were used to set, to configure and to command the real system's components. The simulation results are validated by the experimental results.

According with the system characteristics in our simulation (and also in the real system) the RGV is transporting a single element. But the RGV has a storage capacity of two palettes. In this case the RGV could carry two work pieces but a bottleneck at the intermediate storage stations will occur due to the large machining times of CNC. In these circumstances, using the control system options, we set the values so that the first storage position of RGV accepts only a palette with a work piece and the second accepts only a palette with a part. The waiting time of the RGV, in both circumstances, will be approximately equal to the sum of the CNC machining time and transfer times. In our system the RGV was programmed to wait for the part to be machined.

Industrial systems have AS/RS central storage system with a big capacity, so transporting two elements at the same time on a similar RGV is justified especially if the RGV is part of a diffuse system with more than two work points, with more transfer systems and more intermediate storage systems. In such an industrial system the RGV can carry elements on complex trajectories at multiple work points. Moreover, in such systems with a high storage capacity with a complex buffer space allocation $[11,12]$ RGVs can be used successfully.

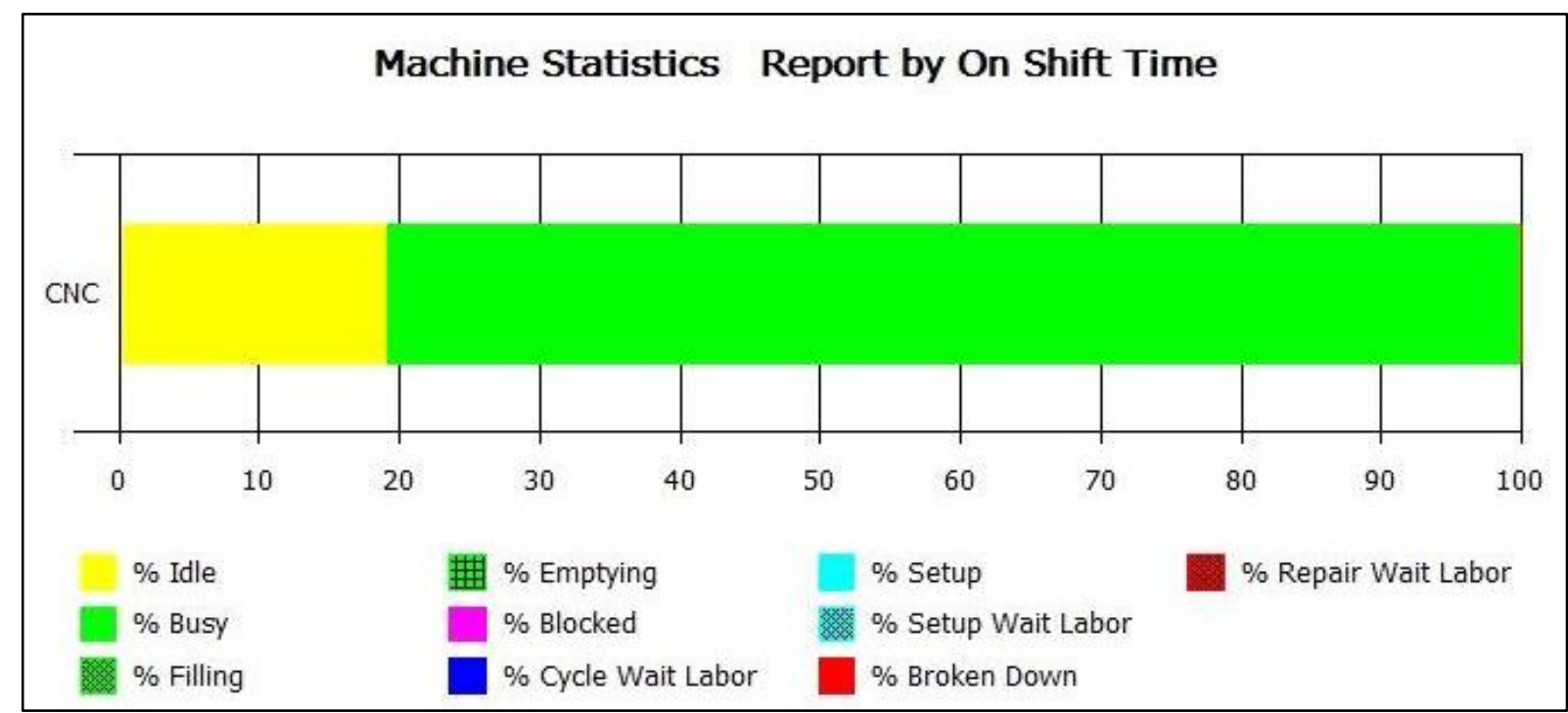

Figure 11: Report regarding the activity of CNC machine after system optimization. 


\section{CONCLUSION AND FUTURE WORK}

Developing an AS/RS educational platform from scratch was a challenge, albeit a chance to test our CAD competencies and results on an educational prototype. In order to adapt this platform to various manufacturing configurations we used simulation on a virtual model of the platform to determine the required parameters for different types of work points and to adjust the part waiting times and the work piece waiting times to be as lower as possible. After the system modelling, the simulation of the system architecture confirmed the system productivity, based on the proposed structural elements parameterization. Also the simulation allowed identifying and eliminating the bottlenecks, long waiting times and ways of improving the system configuration for productivity increase. This virtual model of the platform could be used for diagnose and optimization of different modular manufacturing architectures where the platform could be integrated. For example, in our future research we plan to use the virtual model of the platform to configure a system that meets the simulated system version described in this paper by moving the educational platform to be in the same system with a CNC and a robot. We consider very important to use this digital model to asses various scenarios by simulation and to find the best parametric configuration for all the elements each time before adapting the platform to a new manufacturing facility.

\section{REFERENCES}

[1] Kumar, S. A.; Suresh N. (2009). Production and Operations Management, New Age International Pvt Ltd Publishers, New Delhi

[2] MHI. Automated Storage and Retrieval Systems, from http://www.mhi.org/fundamentals/ automated-storage, accessed on 18-11-2014

[3] Roodbergen, K. J.; Vis, I. F.A. (2009). A survey of literature on automated storage and retrieval systems, European Journal of Operational Research, Vol. 194, No. 2, 343-362, doi:10.1016/j.ejor.2008.01.038

[4] Groover, M. P. (2007). Automation, Production Systems, and Computer-Integrated Manufacturing, $3^{\text {rd }}$ edition, Prentice Hall, Upper Saddle River

[5] Lerher, T.; Ekren, Y. B.; Sari, Z.; Rosi, B. (2015). Simulation analysis of shuttle based storage and retrieval systems, International Journal of Simulation Modelling, Vol. 14, No. 1, 48-59, doi:10.2507/IJSIMM14(1)5.281

[6] Groover, M. P. (2012). Fundamentals of Modern Manufacturing: Materials, Processes, and Systems, John Wiley \& Sons, Hoboken

[7] Cotet, C. E.; Popa, C. L.; Anghel, F. (2009). Manufacturing architecture design using discrete material flow management, International Journal of Simulation Modelling, Vol. 8, No. 4, 206214, doi:10.2507/IJSIMM08(4)3.144

[8] Ayadi, M.; Costa Affonso, R.; Cheutet, V.; Masmoudi, F.; Riviere, A.; Haddar, M. (2013). Conceptual model for management of digital factory simulation information, International Journal of Simulation Modelling, Vol. 12, No. 2, 107-119, doi:10.2507/IJSIMM12(2)4.233

[9] Wang, C.; Liu, X.-B.; Zhao, G.-Z.; Chin, K. O. (2014). Multi-objective integrated production planning model and simulation constrained doubly by resources and materials, International Journal of Simulation Modelling, Vol. 13, No. 2, 243-254, doi:10.2507/IJSIMM13(2)CO10

[10] Debevec, M.; Simic, M.; Herakovic, N. (2014). Virtual factory as an advanced approach for production process optimization, International Journal of Simulation Modelling, Vol. 13, No. 1, 66-78, doi:10.2507/IJSIMM13(1)6.260

[11] Erkan, T. E.; Can, G. F. (2014). Selecting the best warehouse data collecting system by using AHP and FAHP methods, Technical Gazette, Vol. 21, No. 1, 87-93

[12] Bekker, J. (2013). Multi-objective buffer space allocation with the cross-entropy method. International Journal of Simulation Modelling, Vol. 12, No. 1, 50-61, doi:10.2507/ IJSIMM12(1)5.228 